\title{
Scheduling multiple divisible loads on a linear processor network
}

\author{
Matthieu Gallet, Yves Robert, and Frédéric Vivien \\ LIP, CNRS-ENS Lyon-INRIA-UCBL \\ École normale supérieure de Lyon, France \\ \{Matthieu.Gallet, Yves.Robert, Frederic.Vivien\}@ens-lyon.fr
}

\begin{abstract}
Min, Veeravalli, and Barlas have recently proposed strategies to minimize the overall execution time of one or several divisible loads on a heterogeneous linear network, using one or more installments [18, 19]. We show on a very simple example that their approach does not always produce a solution and that, when it does, the solution is often suboptimal. We also show how to find an optimal scheduling for any instance, once the number of installments per load is given. Then, we formally prove that any optimal schedule has an infinite number of installments under a linear cost model as the one assumed in [18, 19]. Such a cost model cannot be used to design practical multi-installment strategies. Finally, through extensive simulations we confirmed that the best solution is always produced by the linear programming approach.
\end{abstract}

\section{Introduction}

Efficiently scheduling the tasks of a parallel application onto the resources of a distributed computing platform is critical for achieving high performance. This scheduling problem has been studied for a variety of application models. Some popular models consider a set of independent tasks without task synchronization nor inter-task communications. Among these models some focus on the case in which the number of tasks and the task sizes can be chosen arbitrarily. This corresponds to the case when the application consists of an amount of computation, or load, that can be arbitrarily divided into any number of independent pieces of arbitrary sizes. This corresponds to a perfectly parallel job: any sub-task can itself be processed in parallel, and on any number of workers. In practice, this model is an approximation of an application that consists of (very) large numbers of identical, low-granularity computations. This divisible load model has been widely studied in the last several years, and Divisible Load Theory (DLT) has been popularized by the landmark book written in 1996 by
Bharadwaj, Ghose, Mani and Robertazzi [4]. DLT has been applied to a large spectrum of scientific problems, including linear algebra [6], image processing [11, 14], video and multimedia broadcasting [1, 2], database searching [5], biological pattern-matching [13], and the processing of large distributed files [17].

Divisible load theory provides a practical framework for the mapping of independent tasks onto heterogeneous platforms. From a theoretical standpoint, the success of the divisible load model is mostly due to its analytical tractability. Optimal algorithms and closed-form formulas exist for the simplest instances of the divisible load problem. We are aware of only one NP-completeness result in the DLT [20]. This is in sharp contrast with the theory of task graph scheduling, which abounds in NP-completeness theorems and in inapproximability results.

Several papers in the Divisible Load Theory field consider master-worker platforms [4, 7, 3]. However, in communication-bound situations, a linear network of processors can lead to better performance: on such a platform, several communications can take place simultaneously, thereby enabling a pipelined approach. Recently, Min, Veeravalli, and Barlas have proposed strategies to minimize the overall execution time of one or several divisible loads on a heterogeneous linear network $[18,19]$. Initially, the authors targeted single-installment strategies, that is strategies under which a processor receives in a single communication all its share of the load. But for cases where their approach failed to design single-installment strategies, they also considered multi-installment solutions.

In this paper, we first show on a very simple example (Section 3) that the approach proposed in [19] does not always produce a solution and that, when it does, the solution is often suboptimal. The fundamental flaw of the approach of [19] is that the authors are optimizing the scheduling load by load, instead of attempting a global optimization. The load by load approach is suboptimal and unduly overconstrains the problem.

On the contrary, we show (Section 4) how to find an optimal scheduling for any instance, once the number of in- 
stallments per load is given. In particular, our approach always find the optimal solution in the single-installment case. We also formally prove (Section 5) that under a linear cost model for communication and communication, as in $[18,19]$, an optimal schedule has an infinite number of installments. Such a cost model can therefore not be used to design practical multi-installment strategies. Finally, in Section 6, we report the simulations that we performed in order to assess the actual efficiency of the different approaches. We now start by introducing the framework.

\section{Problem and Notations}

We use a framework similar to that of $[18,19]$. The target architecture is a linear chain of $m$ processors $\left(P_{1}, P_{2}, \ldots, P_{m}\right)$. Processor $P_{i}$ is available from time $\tau_{i}$. It is connected to processor $P_{i+1}$ by the communication link $l_{i}$ (see Figure 1). The target application is composed of $N$ loads (or tasks, or jobs), which are divisible, which means that each load can be split into an arbitrary number of chunks of any size, and these chunks can be processed independently. All the loads are initially available on processor $P_{1}$, which processes a fraction of them and delegates (sends) the remaining fraction to $P_{2}$ in parallel. In turn, $P_{2}$ executes part of the load that it receives from $P_{1}$ and sends the rest to $P_{3}$, and so on along the processor chain. Communications can be overlapped with (independent) computations, but a given processor can be active in at most a single communication at any time-step: sends and receives are serialized (this is the full one-port model).

Since the last processor $P_{m}$ cannot start computing before having received its first message, it is useful for $P_{1}$ to distribute the loads in several installments: the idle time of remote processors in the chain will be reduced due to the fact that communications are smaller in the first steps of the overall execution.

The objective is to minimize the makespan, i.e., the time at which all loads are completed. For the sake of convenience, all notations are summarized in Table 1.

We deal with the general case in which the $n$th load is distributed in $Q_{n}$ installments of different sizes. For the $j$ th installment of load $n$, processor $P_{i}$ takes a fraction $\gamma_{j}^{n}(i)$, and sends the remaining part to the next processor while processing its own fraction.

Loads have different characteristics: load $n$ (with $1 \leq$ $n \leq N)$ is defined by a volume of data $V_{\text {comm }}(n)$ and a quantity of computation $V_{\text {comp }}(n)$. Moreover, processors and links are not identical either. We let $w_{i}$ be the time taken by $P_{i}$ to compute a unit load $(1 \leq i \leq m)$, and $z_{i}$ be the time taken by $P_{i}$ to send a unit load to $P_{i+1}$ (over link $\left.l_{i}, 1 \leq i \leq m-1\right)$. Note that we assume a linear model for computations and communications, as in the original articles $[18,19]$, and as is often the case in divisible load liter-

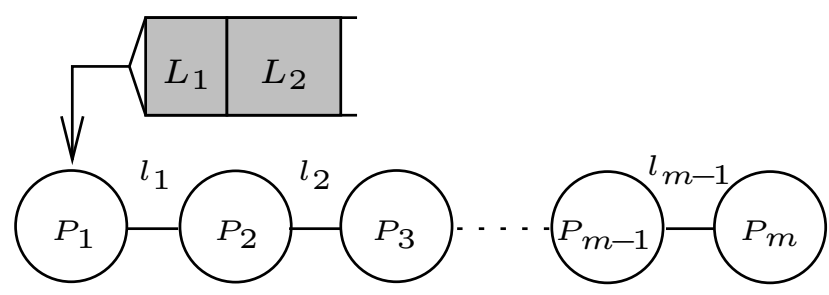

Figure 1. Linear network, with $m$ processors and $m-1$ links.

ature $[16,8]$ (we will discuss this hypothesis in Section 5).

For the $j$ th installment of the $n$th load, let $C_{0 m m_{i, n, j}^{s t a r t}}$ denote the starting time of the communication between $P_{i}$ and $P_{i+1}$, and let $C o m m_{i, n, j}^{e n d}$ denote its completion time; similarly, Comp start denotes the start time of the computation on $P_{i}$ for this installment, and $C o m p_{i, n, j}^{e n d}$ denotes its completion time. Following $[18,19]$, we make the assumption that processor $P_{i}$ sends the relevant fraction of the $j$ th installment of the $n$th load to processor $P_{i+1}$ before it starts to receive another fraction of load from $P_{i-1}$. Similarly, we suppose that the order in which the different application loads are sent is fixed. Although very natural, these assumptions do reduce the solution space, and it might be useful to relax them in some special cases.

\section{Motivating example}

We first recall the algorithms presented in [19]. We then introduce our motivating example and use it to assess the performance of these algorithms.

\subsection{The existing algorithms}

It is often stated that, when scheduling a single load under the divisible load model, in an optimal solution "all participating processors stop computing at the same time instant" [19]. This property has been formally proved for some particular settings $[3,7]$ but is used far more generally and some existing proofs are even flawed (see [7] for examples).

Min, Veeravalli, and Barlas use this optimality principle to build their algorithm. They assume that all processors participate in the processing of each load and all complete simultaneously the processing of any given load. The strict application of this principle leads to what we call the SingleInST algorithm. In order to further optimize the processing of the loads, they force each processor to stay busy from the first time it starts processing a load to the overall completion. When such a solution does not exist 


\begin{tabular}{|ll|}
\hline$m$ & Number of processors in the system. \\
\hline$P_{i}$ & Processor $i$, where $i=1, \ldots, m$. \\
\hline$w_{i}$ & Time taken by processor $P_{i}$ to compute a unit load. \\
\hline$z_{i}$ & Time taken by $P_{i}$ to transmit a unit load to $P_{i+1}$. \\
\hline$\tau_{i}$ & Availability date of $P_{i}$ (time at which it becomes available for processing the loads). \\
\hline$N$ & Total number of loads to process in the system. \\
\hline$Q_{n}$ & Total number of installments for $n$th load. \\
\hline$V_{\text {comm }}(n)$ & Volume of data for $n$th load. \\
\hline$V_{\text {comp }}(n)$ & Volume of computation for $n$th load. \\
\hline$\gamma_{i}^{j}(n)$ & Fraction of $n$th load computed on processor $P_{i}$ during the $j$ th installment. \\
\hline Comm $m_{i, n, j}^{\text {start }}$ & $\begin{array}{l}\text { Start time of communication from processor } P_{i} \text { to processor } P_{i+1} \\
\text { for } j \text { th installment of } n \text {th load. }\end{array}$ \\
\hline Comm $m_{i, n, j}^{\text {end }}$, & $\begin{array}{l}\text { End time of communication from processor } P_{i} \text { to processor } P_{i+1} \\
\text { for } j \text { th installment of } n \text {th load. }\end{array}$ \\
\hline Comp $p_{i, n, j}^{\text {start }}$ & $\begin{array}{l}\text { Start time of computation on processor } P_{i} \\
\text { for } j \text { th installment of } n \text {th load. }\end{array}$ \\
\hline Comp $p_{i, n, j}^{\text {end }}$ & $\begin{array}{l}\text { End time of computation on processor } P_{i} \\
\text { for } j \text { th installment of } n \text {th load. }\end{array}$ \\
\hline
\end{tabular}

Table 1. Summary of notations.

with a single-installment strategy, that is when a processor receives in a single communication all its share of a given load, they resort to multi-installment strategies where each installment is the largest possible satisfying all the constraints (all processors complete simultaneously an installment processing). This defines their main algorithm, that we call MultiInst. The idea is to fully overlap communications by computations (which is obviously not always possible when communications are far more expensive than computations). Both algorithms optimize the schedule load by load, instead of attempting a global optimization.

\subsection{The example}

Our motivating example uses 2 identical processors $P_{1}$ and $P_{2}$ with $w_{1}=w_{2}=\lambda$, and $z_{1}=1$. We consider $N=2$ identical divisible loads to process, with $V_{\text {comm }}(1)=V_{\text {comm }}(2)=1$ and $V_{\text {comp }}(1)=V_{\text {comp }}(2)=$ 1 . Note that when $\lambda$ is large, communications become negligible and each processor is expected to process around half of both loads. But when $\lambda$ is close to 0 , communications are very important, and the solution is not obvious. As both processors have the same computational power, under MultiInst they will process the same fraction of any given installment of any given load, except for the first installment of the first load.

To ease the reading, we only give a short (intuitive) description of the schedules, and we provide the different makespans without justification; all details can be found in the research report [15].

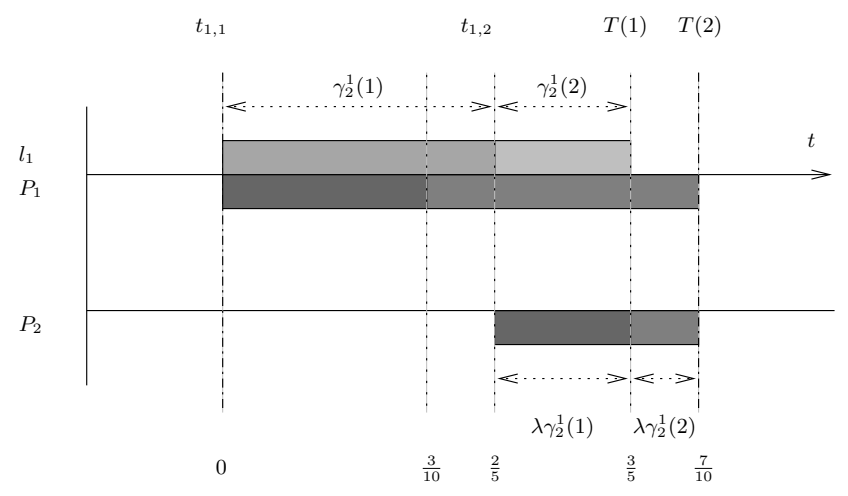

Figure 2. A possible schedule when $\lambda=\frac{1}{2}$.

We first consider a simple schedule which uses a single installment for each load, as illustrated in Figure 2. Processor $P_{1}$ computes a fraction $\gamma_{1}^{1}(1)=\frac{2 \lambda^{2}+1}{2 \lambda^{2}+2 \lambda+1}$ of the first load, and a fraction $\gamma_{1}^{1}(2)=\frac{2 \lambda+1}{2 \lambda^{2}+2 \lambda+1}$ of the second load. Then the second processor computes a fraction $\gamma_{2}^{1}(1)=\frac{2 \lambda}{2 \lambda^{2}+2 \lambda+1}$ of the first load, and a fraction $\gamma_{2}^{1}(2)=$ $\frac{2 \lambda^{2}}{2 \lambda^{2}+2 \lambda+1}$ of the second load. The makespan achieved by this schedule is equal to makespan ${ }_{1}=\frac{2 \lambda\left(\lambda^{2}+\lambda+1\right)}{2 \lambda^{2}+2 \lambda+1}$.

\subsection{Case $\lambda \geq \frac{\sqrt{3}+1}{2}$ : single-installment}

Under the algorithms of [19], $P_{1}$ and $P_{2}$ have to simultaneously complete the processing of their share of the first 


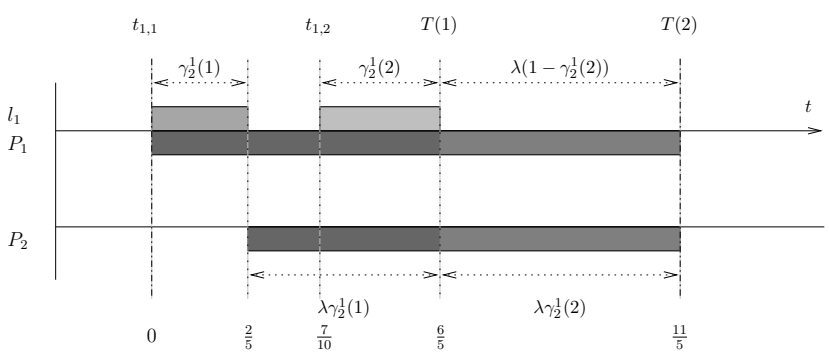

Figure 3. The schedule of [19] for $\lambda=2$.

load. The same holds true for the second load. We are in the one-installment case when $P_{1}$ is fast enough to send the second load to $P_{2}$ while it is computing the first load (hence SingleInst and MultiInst have the same output). This condition writes $\lambda \geq \frac{\sqrt{3}+1}{2} \approx 1.366$. Then, $P_{1}$ processes a fraction $\gamma_{1}^{1}(1)=\frac{\lambda+1}{2 \lambda+1}$ of the first load, and a fraction $\gamma_{1}^{1}(2)=\frac{1}{2}$ of the second one. The makespan achieved by this schedule is makespan $_{2}=\frac{\lambda(4 \lambda+3)}{2(2 \lambda+1)}$.

Comparing both makespans, we have $0 \leq$ makespan $_{2}-$ makespan $_{1} \leq \frac{1}{4}$, the solution of [19] having a strictly larger makespan, except when $\lambda=\frac{\sqrt{3}+1}{2}$. A visual representation of this case is given in Figure 3 for $\lambda=2$.

\subsection{Case $\lambda<\frac{\sqrt{3}+1}{2}$ : multi-installment}

The solution of [19] is a multi-installment strategy when $\lambda<\frac{\sqrt{3}+1}{2}$, i.e., when communications tend to be important compared to computations. More precisely, this case happens when $P_{1}$ does not have enough time to completely send the second load to $P_{2}$ before the end of the computation of the first load on both processors.

The way to proceed in [19] is to send the second load using a multi-installment strategy. $Q_{2}$ denote the number of installments for this second load. We can easily compute the size of each fraction distributed to $P_{1}$ and $P_{2}$. Processor $P_{1}$ has to process a fraction $\gamma_{1}^{1}(1)=\frac{\lambda+1}{2 \lambda+1}$ of the first load, and fractions $\gamma_{1}^{1}(2), \gamma_{1}^{2}(2), \ldots, \gamma_{1}^{Q_{2}}(2)$ of the second one. Moreover, for $1 \leq k<Q_{2}$, due to all the assumptions, we have $\gamma_{1}^{k}(2)=\lambda^{k} \gamma_{2}^{1}(1)$. And for $k=Q_{2}$ (the last installment), we have $\gamma_{1}^{Q_{2}}(2) \leq \lambda^{Q_{2}} \gamma_{2}^{1}(1)$. We can then establish an upper bound on the portion of the second load distributed in $Q_{2}$ installments:

$$
\sum_{k=1}^{Q_{2}}\left(2 \gamma_{1}^{k}(2)\right) \leq 2 \sum_{k=1}^{Q_{2}}\left(\gamma_{2}^{1}(1) \lambda^{k}\right)=\frac{2\left(\lambda^{Q_{2}}-1\right) \lambda^{2}}{2 \lambda^{2}-\lambda-1}
$$

if $\lambda \neq 1$, and $Q_{2}=2$ otherwise. We have three cases to discuss:

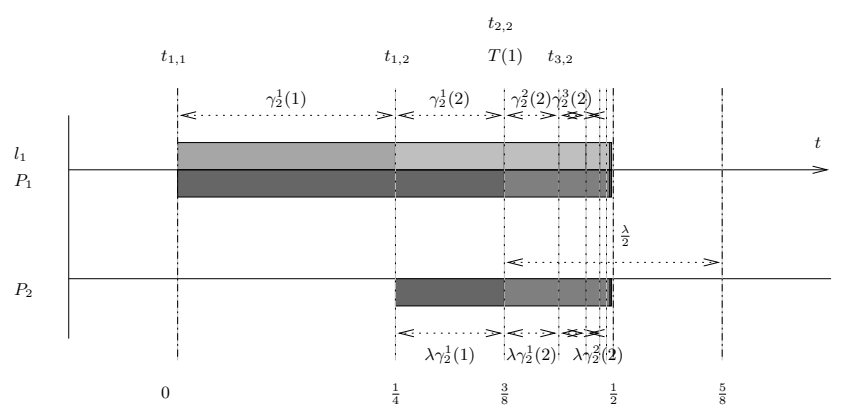

Figure 4. The schedule of [19] for $\lambda=\frac{1}{2}$.

1. $0<\lambda<\frac{\sqrt{17}+1}{8} \approx 0.64$ : Since $\lambda<1$, we can write for any nonnegative integer $Q_{2}$ :

$$
\sum_{k=1}^{Q_{2}}\left(2 \beta_{k}\right)<\sum_{k=1}^{\infty}\left(2 \beta_{i}\right)=\frac{2 \lambda^{2}}{(1-\lambda)(2 \lambda+1)}
$$

$\frac{2 \lambda^{2}}{(1-\lambda)(2 \lambda+1)}<1$ when $\lambda<\frac{\sqrt{17}+1}{8}$. So, an infinite number of installments do not suffice to completely process the second load. In other words, no solution is found in [19] for this case. A visual representation of this case is given in Figure 4 with $\lambda=0.5$.

2. $\lambda=\frac{\sqrt{17}+1}{8}$ : Then $\frac{2 \lambda^{2}}{(1-\lambda)(2 \lambda+1)}=1$, and an infinite number of installments is required to completely process the second load. This solution is unrealistic.

3. $\frac{\sqrt{17}+1}{8}<\lambda<\frac{\sqrt{3}+1}{2}$ : The solution of [19] is then a multi-installment solution which is better than any solution using a single installment per load. (A visual representation of this case is given in Figure 5 with $\lambda=1$.) However this solution may require a very large number of installments and is not always optimal. Indeed, consider the case $\lambda=\frac{3}{4}$. The algorithm of [19] achieves a makespan equal to $\left(1-\gamma_{2}^{1}(1)\right) \lambda+\frac{\lambda}{2}=\frac{9}{10}$. The first load is sent in one installment and the second one is sent in 3 installments, as the number of installments is set in [19] as $Q_{2}=\left\lceil\frac{\ln \left(\frac{4 \lambda^{2}-\lambda-1}{2 \lambda^{2}}\right)}{\ln (\lambda)}\right\rceil$. However, we can come up with a better schedule, maybe suboptimal but still valid, by splitting both loads into two installments, and distributing them as follows:

- Load 1, first round: $P_{1}$ processes 0 unit;

- Load 1, first round: $P_{2}$ processes $\frac{192}{653}$ unit;

- Load 1, second round: $P_{1}$ processes $\frac{317}{653}$ unit;

- Load 1, second round: $P_{2}$ processes $\frac{144}{653}$ unit;

- Load 2, first round: $P_{1}$ processes 0 unit;

- Load 2, first round: $P_{2}$ processes $\frac{108}{653}$ unit;

- Load 2, second round: $P_{1}$ processes $\frac{464}{653}$ unit;

- Load 2, second round: $P_{2}$ processes $\frac{81}{653}$ unit. 


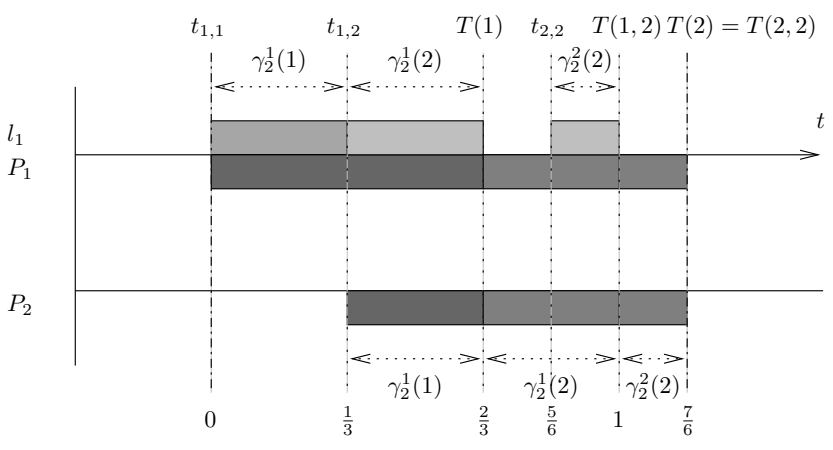

Figure 5. The schedule of [19] for $\lambda=1$.

This scheme gives us a total makespan equal to $\frac{781}{653} \frac{3}{4} \approx 0.897$, which is (slightly) better than 0.9 . This shows that among the schedules having a total number of four installments, the solution of [19] is suboptimal.

\subsection{Conclusion}

Despite its simplicity (two identical processors and two identical loads), out motivating example clearly outlines the limitations of the approach of [19]: this approach does not always return a feasible solution and, when it does, this solution is not always optimal. In the next section, we show how to compute an optimal schedule when dividing each load into any prescribed number of installments. Our simulations will later show that the gap between MuLTIInsT and the optimal schedule can be very important.

\section{Optimal solution}

We now show how to compute an optimal schedule, when dividing each load into any prescribed number of installments. Therefore, when this number of installment is set to 1 for each load (i.e., $Q_{n}=1$, for any $n$ in $[1, N]$ ), the following approach solves the problem originally targeted by Min, Veeravalli, and Barlas.

To build our solution we use a linear programming approach. In fact, we only have to list all the (linear) constraints that must be fulfilled by a schedule, and write that we want to minimize the makespan. All these constraints are captured by the linear program in Figure 6 . The optimality of the solution comes from the fact that the constraints are exactly all the constraints that any schedule must fulfill under the assumptions of Section 2, and a solution to the linear program is obviously always feasible. This linear program simply encodes the following constraints (a constraint has the same number below and in Figure 6):

1. $P_{i}$ cannot start a new communication to $P_{i}$ before the end of the corresponding communication from $P_{i-1}$ to
$P_{i}$,

2. $P_{i}$ cannot start to receive the next installment of the $n$th load before having finished to send the current one to $P_{i+1}$,

3. $P_{i}$ cannot start to receive the first installment of the next load before having finished to send the last installment of the current load to $P_{i+1}$,

4. any transfer has to begin at a nonnegative time,

5. the duration of any transfer is equal to the product of the time taken to transmit a unit load by the volume of data to transfer,

6. processor $P_{i}$ cannot start to compute the $j$ th installment of the $n$th load before having finished to receive the corresponding data,

7. the duration of any computation is equal to the product of the time taken to compute a unit load by the volume of computations,

8. processor $P_{i}$ cannot start to compute the first installment of the next load before it has completed the computation of the last installment of the current load,

9. processor $P_{i}$ cannot start to compute the next installment of a load before it has completed the computation of the current installment of that load,

10. processor $P_{i}$ cannot start to compute the first installment of the first load before its availability date,

11. the portion of a load dedicated to a processor is necessarily nonnegative,

12. any load has to be completely processed,

13. the makespan is no smaller than the completion time of the last installment of the last load on any processor.

Altogether, we have a linear program to be solved over the rationals, hence a solution in polynomial time [10]. In practice, packages like GLPK [9] will return the optimal solution for all reasonable problem sizes. Note that the linear program gives the optimal solution for a prescribed number of installments for each load. In the next section we discuss the problem of the number of installments.

\section{Possible extensions}

Several of the model restrictions can be alleviated. First the model uses uniform machines, meaning that the speed of a processor does not depend on the task that it executes. It is easy to extend the linear program for unrelated parallel machines, introducing $w_{i}^{n}$ to denote the time taken by $P_{i}$ to process a unit load of type $n$. Also, all processors and loads are assumed to be available from the beginning. In our linear program, we have introduced availability dates for processors. The same way, we could have introduced release dates for loads. Furthermore, instead of minimizing the makespan, we could have targeted any other objective function which is an affine combination of the loads completion time and of the problem characteristics, like the av- 


$$
\begin{aligned}
& \forall i<m-1, n \leq N, j \leq Q_{n} \quad \operatorname{Comm}_{i+1, n, j}^{\text {start }} \quad \geq \operatorname{Comm}_{i, n, j}^{\text {end }} \\
& \forall i<m-1, n \leq N, j<Q_{n} \quad \operatorname{Comm}_{i, n, j+1}^{\text {start }} \quad \geq \quad \operatorname{Comm}_{i+1, n, j}^{\text {end }} \\
& \forall i<m-1, n<N \quad \operatorname{Comm}_{i, n+1,1}^{\text {start }} \quad \geq \operatorname{Comm}_{i+1, n, Q_{n}}^{\text {end }} \\
& \forall i \leq m-1, n \leq N, j \leq Q_{n} \quad \operatorname{Comm}_{i, n, j}^{\text {start }} \quad \geq 0 \\
& \forall i \leq m-1, n \leq N, j \leq Q_{n} \quad \operatorname{Comm}_{i, n, j}^{e n d} \\
& \forall i \geq 2, n \leq N, j \leq Q_{n} \\
& \operatorname{Comp}_{i, n, j}^{\text {start }} \\
& \operatorname{Comp}_{i, n, j}^{e n d} \\
& \text { Comp }_{i, n+1,1}^{\text {start }} \\
& \forall i \leq m, n<N \\
& \text { Comp } p_{i, n, j+1}^{\text {start }} \\
& \forall i \leq m \\
& \text { Comp } \text { start }_{i, 1}^{\text {start }} \\
& \forall i \leq m, n \leq N, j \leq Q_{n} \\
& \gamma_{i}^{j}(n) \\
& \forall n \leq N \\
& \sum_{i=1}^{m} \sum_{j=1}^{Q} \gamma_{i}^{j}(n) \\
& =\operatorname{Comm}_{i, n, j}^{\text {start }}+z_{i} V_{\text {comm }}(n) \sum_{k=i+1}^{m} \gamma_{k}^{j}(n) \\
& \geq \operatorname{Comm}_{i, n, j}^{e n d} \\
& =C_{o m p}^{\text {start }}+w_{i, n} \gamma_{i}^{j}(n) V_{\text {calc }}(n) \\
& \geq \operatorname{Comp}_{i, n, Q_{n}}^{\text {end }} \\
& \geq \operatorname{Comp}_{i, n, j}^{\text {end }} \\
& \geq \tau_{i} \\
& \geq 0 \\
& =1 \\
& \forall i \leq m \\
& \text { makespan } \\
& \geq \quad C o m p p_{i, N, Q}^{e n d}
\end{aligned}
$$

Figure 6. The complete linear program.

erage completion time, the maximum or average (weighted) flow, etc.

The formulation of the problem does not allow any piece of the $n^{\prime}$ th load to be processed before the $n$th load is completely processed, if $n^{\prime}>n$. We can easily extend our solution to allow for $N$ rounds of the $N$ loads, each load being still divided into several installments. This would allow to interleave the processing of the different loads.

The divisible load model is linear, which causes major problems for multi-installment approaches. Indeed, once we have a way to find an optimal solution when the number of installments per load is given, the question is: what is the optimal number of installments? Under a linear model for communications and computations, the optimal number of installments is infinite, as the following theorem states:

Theorem 1. Assuming a linear cost model for communications and computations, consider any problem problem with one or more loads and at least two processors. Then, any schedule using a finite number of installments is suboptimal for makespan minimization.

This theorem is proved by building, from any schedule, another schedule with a strictly smaller makespan. The proof is available in the research report [15].

An infinite number of installments obviously does not define a feasible solution. Moreover, in practice, when the number of installments becomes too large, the model is inaccurate, as acknowledged in [4, pp. 224 and 276]. Any communication incurs a startup cost $K$, which we express in bytes. Consider the $n$th load, whose communication volume is $V_{\text {comm }}(n)$ : it is split into $Q_{n}$ installments, and each installment requires $m-1$ communications. The ratio between the actual and estimated communication costs is roughly equal to $\rho=\frac{(m-1) Q_{n} K+V_{\text {comm }}(n)}{V_{\text {comm }}(n)}>1$. Since $K$, $m$, and $V_{\text {comm }}$ are known values, we can choose $Q_{n}$ such that $\rho$ is relatively small, and so such that the model remains valid for the target application. Another, and more accurate solution, would be to introduce latencies in the model, as in [3]. This latter article shows how to design asymptotically optimal multi-installment strategies for star networks. A similar approach could be used for linear networks.

\section{Experiments}

Using simulations, we assess the relative performance of our linear programming approach, of the solutions of [18, 19], and of simpler heuristics. We first describe the experimental protocol and then analyze the results.

Experimental protocol. We use Simgrid [12] to simulate linear processor networks. Schedules are computed by a Perl script, and their validity and theoretical makespan are checked before running them in the simulator.

We study the following algorithms and heuristics:

- The naive heuristic SIMPLE distributes each load in a single installment and proportionally to the processor speeds.

- The strategy for a single load, SingleLoAd, presented by Min and Veeravalli in [18]. For each load, 
we set the time origin to the availability date of the first communication link (in order to prevent communication contentions).

- The SingleInst strategy described in Section 3.1.

- The MultiInst $n$ strategy. This is a slightly modified version of MULTIINST which ensures that a load is not distributed in more than $n$ installments, the $n$th installment distributing all the load remaining work.

- The Heuristic B presented by Min, Veeravalli and Barlas in [19].

- LP $n$ : the solution of our linear program where each load is distributed in $n$ installments.

We measure the relative performance of each heuristic on each instance: we divide the makespan obtained by a given heuristic on a given instance by the smallest makespan obtained, on that instance, among all heuristics. Considering the relative performance enables us to obtain meaningful statistics among instances with very different makespans.

Instances. We emulate a heterogeneous linear network with $m=10$ processors. We consider two distribution types for processing powers: homogeneous where each processor $P_{i}$ has a processing power $\frac{1}{w_{i}}=100$ MFLOPS, and heterogeneous where processing powers are uniformly picked between 10 and 100 MFLOPS. Communication link $l_{i}$ has a speed $\frac{1}{z_{i}}$ uniformly chosen between 10 $\mathrm{Mb} / \mathrm{s}$ and $100 \mathrm{Mb} / \mathrm{s}$. Latencies are between $0.1 \mathrm{~ms}$ (for the fastest links) and $1 \mathrm{~ms}$ (for the slowest ones). For homogeneous and heterogeneous platforms, simulation tasks have their computation volumes either all uniformly distributed between 6 GFLOPS and 4 TFLOPS, or all uniformly distributed between 6 and 60 GFLOPS. For each combination of processing power distribution and task size, we fix the communication to computation volume of all tasks to either $0.01,0.05,0.1,0.5,1.0,5 ., 10,50$, or 100 (bytes per FLOPS). Each instance contains 50 loads. Finally, we randomly built 100 instances per combination of the different parameters, hence a total of 3,600 instances simulated and reported in Table 2. The code and the experimental results can be downloaded from: http: //graal.ens-lyon.fr/ mgallet/downloads / DivisibleLoadsLinearNetwork. tar.gz.

We fixed an upper-bound for the number of installments per load used by the different heuristics : MULtiInst to either 100 or 300 , SINGLELOAD to 100 , and LP $n$ to either $1,2,3$, or 6 .

Discussions of the results. We first remark that the linear program approach always reaches the best makespan. LP 1, LP 2, LP 3, and LP 6 achieve equivalent performance, always less than 5\%o away from the optimal. This may seem counter-intuitive but can be readily explained: multi-installment strategies mainly reduce the idle time in-

\begin{tabular}{|l|r|r|r|}
\hline \multicolumn{1}{|c|}{ Heuristic } & \multicolumn{1}{c|}{ Average } & \multicolumn{1}{c|}{ Std dev. } & \multicolumn{1}{c|}{ Max } \\
\hline SIMPLE & 1150.428871 & 1587.291171 & 8385.941631 \\
\hline SINGLELOAD 100 & 1462.658417 & 2001.397914 & 10714.417531 \\
\hline SINGLEINST & 1.063072 & 0.080003 & 1.523246 \\
\hline MULTIINST 100 & 1.139619 & 0.184372 & 1.987124 \\
\hline MULTIINST 300 & 1.139628 & 0.184385 & 1.987124 \\
\hline HEURISTIC B & 1.132682 & 0.174658 & 2.018659 \\
\hline LP 1 & 1.000472 & 0.000850 & 1.004988 \\
\hline LP 2 & 1.000049 & 0.000096 & 1.001967 \\
\hline LP 3 & 1.000024 & 0.000047 & 1.000981 \\
\hline LP 6 & 1.000000 & 0.000000 & 1.000013 \\
\hline
\end{tabular}

Table 2. Summary of results.

curred on each processor before it starts processing the first task, and the room for improvement is thus quite small in our batches of 50 tasks. The strict one-port communication model forbids the overlapping of some communications due to different installments, and further limits the room for performance enhancement. Except in some peculiar cases, distributing the loads in multi-installments do not induce significant gains. In very special cases, LP 6 does not achieve the best performance during the simulations, but this fact can be explained by the latencies existing in simulations.

The bad performance of SIMPLE, which can have makespans 8000 greater than the optimal, justify the use of sophisticated scheduling strategies. SINGLEINST has tremendously better performance than SINGLELOAD as it far better takes into account communication link availabilities: the huge difference of performance is due to the instances with expensive communications. SINGLEInst achieves very good average performance, within $6 \%$ of the optimal. It also achieves significantly better performance than MultiInst, and Heuristic B. This may also be due to the fact that multi-installment strategies are not efficient in our experimental context. The slight difference performance between MultiInst 100 and MultiInst 300 shows that MultiInst sometimes uses a large amount of installments for an insignificant negative gain (certainly due to the latencies). When communication links are slow and when computations dominate communications, MULTIINST and HEURISTIC B can have makespans 98\% higher than the optimal.

\section{Conclusion}

We have shown that a linear programming approach allows to solve all instances of the scheduling problem addressed in $[18,19]$. In contrast, the original approach was providing a solution only for particular problem instances. Moreover, the linear programming approach returns an optimal solution for any number of installments, while the orig- 
inal approach was empirically limited to very special strategies, and was often sub-optimal.

Intuitively, the solution of [19] is less efficient than the schedule of Section 3.2 because it aims at locally optimizing the makespan for the first load, and then optimizing the makespan for the second one, and so on, instead of directly searching for a global optimum. We were not able to provide closed-form expressions characterizing optimal solutions, but owing to the power of linear programming, we were able to derive an optimal schedule for any problem instance. We validated this approach through simulations which confirmed that the best solution is always produced by the linear programming approach, while solutions of [19] can be far away from the optimal. The simulations also show that, in our settings, the multi-installment strategies rarely lead to significant gains.

\section{References}

[1] D. Altilar and Y. Paker. An optimal scheduling algorithm for parallel video processing. In IEEE Int. Conference on Multimedia Computing and Systems. IEEE Computer Society Press, 1998.

[2] D. Altilar and Y. Paker. Optimal scheduling algorithms for communication constrained parallel processing. In Euro-Par 2002, LNCS 2400, pages 197206. Springer Verlag, 2002.

[3] O. Beaumont, H. Casanova, A. Legrand, Y. Robert, and Y. Yang. Scheduling divisible loads on star and tree networks: results and open problems. IEEE Trans. Parallel Distributed Systems, 16(3):207-218, 2005.

[4] V. Bharadwaj, D. Ghose, V. Mani, and T. Robertazzi. Scheduling Divisible Loads in Parallel and Distributed Systems. IEEE Computer Society Press, 1996.

[5] J. Blazewicz, M. Drozdowski, and M. Markiewicz. Divisible task scheduling - concept and verification. Parallel Computing, 25:87-98, 1999.

[6] S. Chan, V. Bharadwaj, and D. Ghose. Large matrixvector products on distributed bus networks with communication delays using the divisible load paradigm: performance and simulation. Mathematics and Computers in Simulation, 58:71-92, 2001.

[7] S. Genaud, A. Giersch, and F. Vivien. Load-balancing scatter operations for grid computing. Parallel Computing, 30(8):923-946, 2004.

[8] D. Ghose and T. Robertazzi, editors. Special issue on Divisible Load Scheduling. Cluster Computing, 6, 1, 2003.
[9] GLPK: GNU Linear Programming Kit. http:// www. gnu.org/software/glpk/.

[10] N. Karmarkar. A new polynomial-time algorithm for linear programming. In Proceedings of the 16th ACM symposium on Theory of Computing (STOC'84), pages 302-311. ACM Press, 1984.

[11] C. Lee and M. Hamdi. Parallel image processing applications on a network of workstations. Parallel Computing, 21:137-160, 1995.

[12] A. Legrand, L.Marchal, and H. Casanova. Scheduling Distributed Applications: The SIMGRID Simulation Framework. In Proceedings of CCGrid'03, pages 138-145, May 2003.

[13] A. Legrand, A. Su, and F. Vivien. Minimizing the stretch when scheduling flows of biological requests. In Proceedings of SPAA '06, pages 103-112. ACM Press, 2006.

[14] X. Li, V. Bharadwaj, and C. Ko. Distributed image processing on a network of workstations. Int. J. Computers and Applications (ACTA Press), 25(2):1-10, 2003.

[15] F. V. Matthieu Gallet, Yves Robert. Comments on "design and performance evaluation of load distribution strategies for multiple loads on heterogeneous linear daisy chain networks". Research report RR6123, INRIA, 2007. http://hal.inria.fr/ inria-00130294.

[16] T. Robertazzi. Ten reasons to use divisible load theory. IEEE Computer, 36(5):63-68, 2003.

[17] R. Wang, A. Krishnamurthy, R. Martin, T. Anderson, and D. Culler. Modeling communication pipeline latency. In Measurement and Modeling of Computer Systems (SIGMETRICS'98), pages 22-32. ACM Press, 1998.

[18] H. M. Wong and B. Veeravalli. Scheduling divisible loads on heterogeneous linear daisy chain networks with arbitrary processor release times. IEEE Trans. Parallel Distributed Systems, 15(3):273-288, 2004.

[19] H. M. Wong, B. Veeravalli, and G. Barlas. Design and performance evaluation of load distribution strategies for multiple divisible loads on heterogeneous linear daisy chain networks. J. Parallel Distributed Computing, 65(12):1558-1577, 2005.

[20] Y. Yang, H. Casanova, M. Drozdowski, M. Lawenda, and A. Legrand. On the complexity of multiround divisible load scheduling. Research report RR6096, INRIA, 2007. http://hal.inria.fr/ inria-00123711. 